veins of the thigh and readily traced into their smaller tributaries. The presence of this thrombus accounts for the paralysis of the limb and the watery semi-transparent whiteness of the foot. The umbilical vein and arteries presented nothing unusual; liver and spleen large and filled with blood; lungs congested; pericardial sac filed with straw-colored fluid; no evidence of cardiac inflammation. Veins of the brain and its membranes contained dark blood but no thrombi. Ureters and urethra patent and normal; bladder empty; stomach and intestines normal; thyroid and thymus glands normal. Perirenal fat absent or nearly so, while subcutaneous fat was present in a very small degree. Microscopic sections of the kidney have been kindly made for me by Dr. Latham, who will present them for the inspection of the Society. They show the changes of a subacute nephritis. The question that has pre. sented itself to my mind in considering the cause of renal disease in this case is, Was the disease due to septic infection, and, if so, how did it occur? The mother was unhealthy, as her history shows. She was considered an unclean case and extra precautions were taken against further infection. She was iso. lated, during the lying-in, to the extent of being assigned to the care of one nurse who cared for her and her child only. I can not disabuse my mind of the idea that the infective matter still remained somewhere in the parturient canal and that infection took place through the umbilical cord.

Note.-Since I read this paper I have seen two infants, hospital cases, in which I made the diagnosis of renal inflammation, acute; one a boy, the other a girl. Both infants presented symptoms similar to those described in the case reported, but not so severe, as both babies recovered. The dryness of the skin, its cracking in the creases of the flexures and the temperature were nearly the same as in report, except that recovery began when temperature reached normal. In the case of the baby girl we obtained enough urine to test for albumin and found it abundant, but not enough to determine quantity per volume. Both children were very ill. I expected both to die, especially the girl, as she was worse than the boy. The mother of the little girl was not so well generally as the other, and at one time, prior to delivery, had a small amount of albumin in urine. The other mother seemed quite well. Both had a normal lyingin and left the hospital well, the babies still showing the effects of their illness but bidding fair for full recovery. Both babies showed stigmata of faulty development in the bones of the head and the ears. Mothers presented no similar signs.

DISCUSSION.

Dr. G. H. Weaver-Dr. L. Emmett Hall, of New York, has written two articles on primary nephritis in infants. The first appeared in 1887 ; at that time he had collected from his own practice and that of his friends during eighteen months, eight cases of what he called primary nephritis in infants, whose ages varied from 2 days to 2 years. From the literature he collected eleven more, making nineteen cases. He concluded that primary nephritis in infants is not a rare disease, but that it occurs quite frequently, although not usually recognized. Five years later, in 1892, he reported two additional cases, being all that he had seen in five years, so he then modified his first conclusion, and says that primary nephritis in infants is rare and occurs infrequently.

The symptoms he described in this condition are as follows: Continuous fever of an irregular type, generally quite high; dullness and apathy with no focal symptoms of brain disease; marked anemia without emaciation, moderate gastro-intestinal disturbance in the early part, usually vomiting later and a tendency to diarrhea ; and later a tendency to bronchitis. These cases all emphasize the fact that in cases of irregular fever in infants for which there is no apparent cause the urine should be examined, and that in case of convulsions in infants the urine should always be examined. In only a few cases was there marked diminution in the amount of urine observable. Dr. Root's case, it seems to me, might possibly belong in this class, the disease of the kidney not having been caused by infection through the umbilical vessels. Changes in the liver would have been expected and changes in the vessels themselves if the infection had taken place in this way.

Dr. Roor-The temperature in this case was high and during the last two or three days irregular, as Dr. Weaver says, but there was a marked decrease in the flow of urine. No examination of the urine was made, for the reason that we could not secure a sufficient quantity of it

DR. EDwin HAMILL--In regard to the last point mentioned by Dr. Root, that of securing a sample of urine from a baby, by placing a flat sponge over the genitals, one may succeed very often in securing urine, without resorting to the use of the catheter.

\section{A CASE OF NEPHRECTOMY FOR SARCOMA OF THE KIDNEY.}

Read before the Chlcago Pathological Society, Jan. 14, 1895. BY D. W. GRAHAM, M.D.

CHICAgO.

This sarcoma was removed from a child 18 months old. There was no history of injury or acute disease. About six months before the operation a tumor was discovered in the child's right side, which gradually enlarged. I saw the case the last week in September and told the parents I would operate if they desired it but did not offer them any encouragement. They declined the operation at that time but returned the middle of November with the request that I operate, even if the child died. I operated with a kind of protest, they knowing all the dangers.

At the time of operation the tumor filled the entire side of the abdomen extending down into the pelvis; it was of enormous size compared with the size of the child. I operated by what is called Langenbeck's method. The incision through the wall is made in the linea semilunaris. The colon is pushed toward the middle line, the peritoneum over the kidney and tumor is cut through and stripped off with the fingers, the tumor being thus enucleated. The pedicle consisting of the usual vessels and the ureter is isolated, the tumor freed from behind and the vessels secured. I will pass this tumor around. The kidney substance is at the upper end, the capsule seems to have been ruptured and the whole mass protruded downward. The mass seems to have come out as a fungus at the pelvis of the kidney, shooting out toward the mid-line of the body, and even surrounding the vena cava. The ureter ran across the tumor. The pedicle was secured in the usual way without great loss of blood. There was a good deal of hemorrhage but it came from the bed of the tumor, there being a good deal of venous oozing which could not be checked readily. For the age and size of the patient a good deal of blood was lost, although the absolute amount was small. The patient lived about four hours and died of shock. A diagnosis of sarcoma of the kidney was made before the operation, and the microscopic examination shows it to be sarcoma of the round-celled variety. The diagnosis was based chiefly on the age of the patient. There is no way of determining beforehand from the clini. cal symptoms whether a tumor of the kidney is carcinoma or sarcoma, but we know that malignant tumors of the kidney in young children are relatively much more frequently sarcomata rather than carcinomata. Dr. Bishop will describe the sections he has made.

MICROSCOPIC EXAMINATION BY DR. D. D. BISHOP.

Pieces were taken from the portion of the specimen which projects above the capsular-like structure and ex- 
amined microscopically. In the main we might say the structure is that of a round-celled sarcoma. The cells are rather large, have large nuclei, some are elongated having the appearance of a so-called oat-shape cell which we see in some tumors. The structure is traversed in a gross way by broad bands of fibrous tissue showing well formed connective tissue in their interiors, but as we pass from the interior of these bands toward the tumor itself, the cells become of the same character as the cells in the tumor proper, indicating that perhaps these have developed by fibrillation and condensation of the tumor structure. There are numerous small blood vessels and these show a distinct endothelial lining and a slight amount of connective tissue wall. From the number of blood vessels and the fact that the round cells are arranged rather in groups about these vessels, one might think it likely that the tumor had its origin in the vascular system, and I consider that perhaps this was the fact, because in other portions there are distinct trabeculated areas in which there are rather large spaces and the trabecular walls are lined with endothelium, very much like other angiomata, so we may consider that it is probably an angio sarcoma. It is interesting to note in all the sections prepared and examined, about twenty-five in number, that in no part of the kidney proper was there evidence of invasion of the sarcoma. The kidney was apparently pushed to one side by the process. The sections from the kidney proper, taken from the capsular-like portion show simply an atrophic condition of the glomeruli, some slight thickening of the walls, compression of the renal tubules and increase in the interstitial tissue. Considering the apparently profound changes and the size of the entire structure the kidney is really very little altered. The renal changes would not be considered very grave if found in a kidney not compressed by a tumor of this sort. In no portion of the structure were there found any of the striated muscle cells which are said to occur of ten in some congenital sarcomata of the kidney, the so-called myo-sarcoma. So it seems that we have not to deal with that variety of tumor but a round-celled sarcoma probably developed in the course of the blood vessels.

Discussion.

Dr. GraHAM-Many of these cases of sarcoma in young children are accounted congenital. There was a year of life elapsing between the time of birth and the time this tumor was discovered. There was nothing in the history or appearance of things that would lead us to believe that this was not a congenital tumor and $I$ am inclined to think the disease was present at birth.

Dr. E. R. LE CoUNT-In reference to congenital sarcoma of the kidney of a malignant character, as reported by Dr. Graham, it is very probable that they have their origin in the remains of the Wolffian bodies, while with tumors that appear later in life there is a tendency to consider their origin as having some connection with remnants of the supernumerary adrenal glands.

Dr. Brshop-Dr. Le Count speaks of the Wolffian bodies in connection with these tumors, and I ought to add that in some of the sections are tubular-like structures lined with columnar celled epithelium, suggesting that they are the remnants of the ducts of the Wolffian body, and should not be overlooked, but they were so few in number and had no apparent relation to the tumor so were not considered as having any etiologic significance. The tumor perhaps, but not necessarily, occurred from that structure. I did not mean to say that this tumor was not in my opinion congenital. I simply stated that the striated muscle cells which are often present in such tumors were not present in this; a congenital tumor may be round-celled as well as any other variety.

\section{A CASE OF TUBAL PREGNANCY.}

Rear before the Chicago Pathological Society, Jan. 14, 1895

\section{BY BERTHA YAN HOOSEN.}

CIrrCAGio.

Mrs. S., aged 22, married three and one-half years had a miscarriage after being married five months. Thre months after the miscarriage had an attack of pelvic inflammation, and a pyosalpinx on the right side was diagnosed by Dr. Bayard Holmes, who was caring for the patient at this time. She was confined to bed for six months at this time, and spent six weeks of the time in Mercy Hospital. She improved while under hospital treatment and was told that she might fully recover if she continued the hot douches after leaving the hospital. Sept. 19, 1894, two years later, the patient came to Dr. Hickey-Carr complaining of nausea and with the history of having missed two monthlies. Examination of the pelvis showed the uterus enlarged and a mass was felt on the right side. Liquid diet was ordered; September 23, the patient was feeling well and took an eight-mile ride on the cable car. September 24, the following day at 3 A.M., she was seized with pain in the pelvis and a neighboring physician was called in. He gave her three doses of codeia $\frac{1}{3}$ gr. each. At 8 A.M. on same morning Dr. Carr was called and found her still in pain; slight hemorrhage from the uterus but no sign of membranes, pelvic tenderness and the mass on the right more distinctly outlined.

September 24, 25, 26, patient kept in bed on liquid diet without anodynes and efforts made to move the bowels with calomel, castor oil, olive oil, glycerin and water enemas but with no success. Up to and including September 26 , the fourth day of the attack, the pulse was 90 to 96 , temperature 99 to $99 \frac{1}{2}$, and the pain constant. September 27, at 4 A.M., the patient had a third violent attack of pain and by 9 A.M. the pulse had risen to 120 , temperature 100 . Dr. Carr was obliged to leave the patient to attend court, and did not see her until 2 o'clock in the afternoon. At 1 o'clock she had been seized with pain and went into collapse. When Dr. Carr saw her at 2 P.M. there was dullness in the lower segment of the abdomen and the mass of the right was very indefinite. I saw the patient two hours later in consultation with Dr. Carr. The temperature was then 100 , and the radial pulse 160 . The patient was blanched and appeared to be sinking rapidly.

Ectopic gestation was suspected and an exploratory operation was decided upon; at $6: 30$ p.M. the patient was taken to the Chicago Hospital, a distance of four blocks from her home. Dr. F. Byron Robinson saw the patient at 8 P.M., confirmed the diagnosis and at 8:15 with the assistance and consultation of Dr. Robinson, I opened the abdominal cavity, the lower portion of which was packed with loose black clots of blood. These with a quantity of liquid blood were turned out, the appendages on the right side were removed, the cavity freely irrigated, glass drainage tube inserted and wound closed with silkworm gut. At 9 P.M., a few minutes after the operation the pulse was 152, temperature 101.4. From this time the pulse dropped until on the seventh day it did not go above 100. On the fourth day the decidua was expelled entire. Drainage removed on the sixth day and stitches tightened; the wound closed by figure 8 ligature and the patient left the hospital on the fourteenth day. I saw her last week and she asserted she had not been so well as now, since her marriage. Since the operation she has gained in weight, menstruates regularly, and is at all times perfectly free from pain.

The specimen is a very typical one and shows what a violent effort was made to accomplish tubal abortion during the four days before tubal rupture took place.

Such a mishap as this illustrates, fortunately does not occur every day, because nature has practically castrated such women by closing the tubes, or so modifying the secretion that conception can not take 